\title{
CiliarMove: new software for evaluating ciliary beat frequency helps find novel mutations by a Portuguese multidisciplinary team on primary ciliary dyskinesia
}

\author{
Pedro Sampaio ${ }^{1}$, Mónica Ferro da Silva ${ }^{1}$, Inês Vale², Mónica Roxo-Rosa ${ }^{1}$, \\ Andreia Pinto (10) ${ }^{1}$, Carolina Constant $\mathbb{0}^{3}$, Luisa Pereira ${ }^{3}$, Carla M. Quintão ${ }^{2,4}$ and \\ Susana S. Lopes $\mathbb{1}^{1}$
}

Affiliations: ${ }^{1}$ iNOVA4HEALTH, CEDOC, NOVA Medical School, Faculdade de Ciências Médicas, Universidade Nova de Lisboa, Lisboa, Portugal. '2Departamento de Física, Faculdade de Ciências e Tecnologia da Universidade Nova de Lisboa, Monte da Caparica, Caparica, Portugal. ${ }^{3}$ Unidade de Pneumologia Pediátrica, Departamento de Pediatria, Hospital de Santa Maria CHLN, Centro Académico de Medicina de Lisboa, Lisboa, Portugal. ${ }^{4}$ NOVA Laboratório de Instrumentação, Engenharia Biomédica e Física da Radiação (LIBPhys-UNL), Lisboa, Portugal.

Correspondence: Susana Lopes, Cilia Regulation and Disease lab (1.18), CEDOC - Centro de Estudos de Doenças Crónicas, NOVA Medical School/Faculdade de Ciências Médicas, Universidade Nova de Lisboa CEDOC II, Rua Câmara Pestana no 6, 6-A, 1150-082 Lisboa, Portugal. E-mail: susana.lopesđanms.unl.pt

ABSTRACT Evaluation of ciliary beat frequency (CBF) performed by high-speed videomicroscopy analysis (HVMA) is one of the techniques required for the correct diagnosis of primary ciliary dyskinesia (PCD). Currently, due to lack of open-source software, this technique is widely performed by visually counting the ciliary beatings per a given time-window. Our aim was to generate open-source, fast and intuitive software for evaluating CBF, validated in Portuguese PCD patients and healthy volunteers.

Nasal brushings collected from 17 adult healthy volunteers and 34 PCD-referred subjects were recorded using HVMA. Evaluation of CBF was compared by two different methodologies: the new semi-automated computer software CiliarMove and the manual observation method using slow-motion movies. Clinical history, nasal nitric oxide and transmission electron microscopy were performed for diagnosis of PCD in the patient group. Genetic analysis was performed in a subset $(n=8)$ of suspected PCD patients.

The correlation coefficient between the two methods was $\mathrm{R}^{2}=0.9895$. The interval of $\mathrm{CBF}$ values obtained from the healthy control group $(n=17)$ was $6.18-9.17 \mathrm{~Hz}$ at $25^{\circ} \mathrm{C}$. In the PCD-excluded group $(\mathrm{n}=16)$, CBF ranged from 6.84 to $10.93 \mathrm{~Hz}$ and in the PCD group $(\mathrm{n}=18)$, CBF ranged from 0 to $14.30 \mathrm{~Hz}$.

We offer an automated open-source programme named CiliarMove, validated by the manual observation method in a healthy volunteer control group, a PCD-excluded group and a PCD-confirmed group. In our hands, comparisons between CBF intervals alone could discern between healthy and PCD groups in $78 \%$ of the cases.

@ERSpublications

New software for easy and faster evaluation of ciliary beat frequency in an unbiased manner validated in three groups: healthy volunteers; patients with confirmed primary ciliary dyskinesia (PCD); and patients with PCD excluded https://bit.ly/2Kvwwes

Cite this article as: Sampaio P, da Silva MF, Vale I, et al. CiliarMove: new software for evaluating ciliary beat frequency helps find novel mutations by a Portuguese multidisciplinary team on primary ciliary dyskinesia. ERJ Open Res 2021; 7: 00792-2020 [https://doi.org/10.1183/23120541.00792-2020].

This article has supplementary material available from openres.ersjournals.com.

Received: 28 Oct 2020 | Accepted after revision: 09 Dec 2020

Copyright $\odot$ ERS 2021. This article is open access and distributed under the terms of the Creative Commons Attribution Non-Commercial Licence 4.0. 


\section{Introduction}

Primary ciliary dyskinesia (PCD; OMIM 244400) is a congenital heterogeneous disorder and prevalence is estimated to be $\sim 1: 10000$ [1], being higher in consanguineous populations [2]. It is thought that the correct prevalence of the disease is unknown because many patients are believed to remain undiagnosed. PCD is characterised by a deficient mucociliary clearance, which is caused by one or a mixture of factors such as lack of motile cilia, uncoordinated ciliary pattern or a total lack of ciliary motion. PCD leads to chronic respiratory infections, and the earlier it is diagnosed the better prognosis the patients will have [3-5]. Its clinical features usually begin at birth with respiratory distress followed by a wet cough in early childhood and evolve to include bronchiectasis and chronic sinusitis. However, PCD is not just a respiratory disease, because half of the PCD cases are associated with situs inversus and heterotaxy; in the latter cases there is a high correlation with congenital heart disease [1]. In addition, male sterility is common because sperm flagella are specialised motile cilia that may also present defective motility [6]. PCD usually follows an autosomal recessive inheritance pattern [7].

Cilia are highly conserved cellular organelles classified according to their axoneme ultrastructure into primary cilia (nonmotile) and motile cilia [8]. The axonemes of motile cilia have an ultrastructure composed of nine peripheral doublets of microtubules with inner and outer dynein arms (IDA and ODA, respectively) surrounding a central pair of microtubules [9]. The axonemal motor dyneins are localised in the IDA and ODA [10]; these specific axonemal dyneins are responsible for the ATP-dependent force that triggers cilia motility. In addition, the nexin-dynein regulatory complex has been identified as the regulator of dynein activity [10]. Cilia motility is assured by the combination of several other structures such as the central pair, radial spokes and the nexin-dynein regulatory complex [11].

PCD is hard to diagnose because of its lack of specific clinical and laboratory signs. However, in the presence of suggestive clinical symptoms there is a clinical diagnostic predictive score for PCD named PICADAR [12]. Therefore, patients can now be readily identified by their general practitioner and referred for further specific testing in specialised centres. PCD diagnosis has no gold-standard reference test and it requires sophisticated different tests from multidisciplinary teams to properly exclude or detect PCD [13], which has motivated a previous review from our team to alert the Portuguese paediatric community [14]. European specialised centres follow diagnostic algorithms, which may differ in the combination of five tests: nasal nitric oxide (nNO), high-speed videomicroscopy analysis (HVMA), transmission electron microscopy (TEM), immunofluorescence and genetic study [15]. Diagnosis is most commonly made through the detection of low nNO; abnormal ciliary beat frequency (CBF) or ciliary beat pattern (CBP) and by ultrastructural defects found on a defined percentage of ciliary axonemes using TEM. To identify the ultrastructural ciliary components and their potential defects, TEM is crucial [9]. Clear ultrastructural defects identified by TEM are enough for a positive diagnosis of PCD [13]. However, genetic variants of PCD that have either subtle alterations or normal ciliary ultrastructure are likely to be missed, accounting for $30 \%$ of all PCD cases [16]. Therefore, TEM is insufficient as a standalone diagnostic test and should be used within a multidisciplinary approach.

To access the CBF and CBP, digital HVMA must be completed. This method offers the only direct readout of ciliary function. Although HVMA was traditionally based on CBF measurement [17], this test has proven only useful in identifying PCD cases that either lead to decreased or increased CBFs [18-20]. STANNARD et al. [21] have demonstrated that the isolated study of CBF results in low sensitivity and specificity levels, leading to a significant number of missed diagnoses. The same study showed that this limitation can be partially resolved with combined analysis of CBP which renders higher positive and negative predictive values in diagnosing PCD.

Recent studies reviewed by RUBBO and LuCAS [1] showed a clear association of beat pattern, ultrastructure defects and genotype. However, CBP analysis requires highly trained technicians to qualitatively differentiate PCD samples from non-PCD samples. Moreover, repeated HVMA after air-liquid interface culture of the biopsied cells is recommended to discard any secondary defects derived from inflammation or infection in the nasal epithelium [13]. This can be considered a limitation, since the construction of this infrastructure adds significant costs to the diagnostic centres. Despite these disadvantages, RuBbo et al. [22] showed that, when using ERS guidelines as reference, HVMA can achieve excellent levels of sensitivity and specificity in diagnosing PCD.

Our study is focused on the digital high-speed videomicroscopy analysis of ciliary motion and aims to validate the new software named CiliarMove developed to evaluate CBF in a semi-automated manner to aid PCD diagnostic teams.

\section{Methods}

\section{Eligibility}

Children with recurrent upper or lower respiratory infections and/or situs abnormalities were referred to our PCD workgroup organised in a multidisciplinary team. Demographic, clinical and diagnostic test data 
were collected. The clinical data were also used to calculate the PICADAR score [12]. Informed consent was obtained for diagnostic tests, after explanation of all procedures to parents and subjects.

After final diagnosis, subjects were subcategorised as a PCD-excluded group, where PCD was ruled out (10 females and six males, age range 3-36 years, mean age 13 years), and a PCD group which included the subjects with a positive diagnosis for the disease ( 11 females and seven males, age range 4-54 years, mean age 16 years). We excluded PCD based on a normal result found in both HVMA (no abnormal CBF and CBP), TEM ( $<20 \%$ abnormal cilia, according to PAPON et al. [9]) and normal nNO values. Positive PCD diagnosis was confirmed according to the European Respiratory Society (ERS) diagnostic guidelines [13], supported by clinical history, PCD-specific features found by TEM (hallmark ciliary ultrastructure defects) and/or genetic testing (biallelic mutations in PCD-causing genes).

Diagnosis was considered extremely unlikely, and therefore applied to the PCD-excluded group, when referred patients presented modest clinical suspicion and had no significant findings by TEM in addition to no defects by HVMA and nNO.

Healthy controls were recruited from the local community. We selected 17 adult nonsmoking volunteers (10 females and seven males, age range 23-43 years, mean age 29 years). We did not exclude volunteers with seasonal allergies, provided they were free of symptoms. None of the volunteers had a respiratory tract infection in the month before the study, although this information was self-reported. This information is relevant, as the recovery of the respiratory epithelium may require $\geqslant 35$ days $[23,24]$. Informed consent was obtained from healthy volunteers according to the NOVA Medical School ethics committee.

\section{Sample collection and preparation for HVMA}

Nasal cells were collected from the inferior turbinate nasal epithelia [13]. Cellular samples adherent to the cytology brush ( $3.1 \mathrm{~mm}$ diameter; MCA Lactona, Loulé, Portugal) were immersed in buffered culture medium 199 (Gibco-Life Technologies, Waltham, MA, USA) to which was added $2.5 \mu \mathrm{L}$ of penicillin-streptomycin 1\% (v/v) (Gibco-Life Technologies). The samples were conserved at temperatures $25-27^{\circ} \mathrm{C}$ during transportation and were then equilibrated at $25^{\circ} \mathrm{C}$ in the lab, monitored by a thermometer next to the microscope, for a minimum of 30 min before HVMA.

Each sample was analysed on a glass slide using silicone grease (Dow Corning Corporation, Midland, MI, USA) to make a small sealed compartment to contain the liquid with the sample avoiding overflowing. Slides were then covered with coverslips and observed with an inverted microscope Nikon Eclipse Ti-U (Tokyo, Japan) with a $100 \times$ objective (1.3 NA) coupled to a high-speed Photron camera FASTCAM MC2 (Tokyo, Japan). The required velocity to perform this analysis was 500 frames per second (fps) as indicated by guidelines [13]. Samples were screened for rotational cilia by performing top-view movies and were carefully screened for the presence of stiffer cilia or cilia with a reduced beat amplitude by performing both top- and lateral-view short movies.

\section{TEM}

Human nasal epithelia samples obtained by nasal brushings were fixed in $2.5 \%(\mathrm{v} / \mathrm{v})$ glutaraldehyde in $0.1 \mathrm{M}$ sodium cacodylate buffer at $4^{\circ} \mathrm{C}$, washed overnight and post-fixed in $1 \%(\mathrm{v} / \mathrm{v})$ osmium tetroxide. After dehydration, the samples were embedded in epoxy resin. Cuts were obtained using a Reichert Supernova ultramicrotome (Leica Microsystems, Wetzlar, Germany). The semi-thin sections $(500 \mathrm{~nm})$ were stained with toluidine blue and the areas of interest were selected. The ultrathin sections $(80 \mathrm{~nm})$ were contrasted with $2 \%$ uranyl acetate in $70 \%$ methanol and lead citrate, and visualised in a TEM Hitachi H-7000 (Hitachi - Science \& Technology, Tokyo, Japan) at $100 \mathrm{kV}$. All cross-sectional cilia were analysed. The ultrastructural examination was performed by a quantitative method in line with the recent international guidelines for PCD [25].

\section{Genetics}

For eight patients with a very likely PCD diagnosis, based on the clinical history and the three techniques used, a genetic study was performed. Biallelic mutations in PCD-causing genes were identified in all eight subjects, of whom four showed homozygous mutations and four showed compound heterozygous mutations (two had been referred previously by FASSAD et al. [26]). The genetic study was performed by collecting blood from prospective patients. Genomic DNA was extracted and whole-exome sequencing was performed using next-generation sequencing subcontracted to GenoMed (Lisbon, Portugal); this company offers an updated clinical panel of genes for PCD diagnostic purposes.

\section{High-speed videomicroscopy}

10 separate clusters of cells from each subject were filmed at $500 \mathrm{fps}$ and data were analysed posteriorly using two different methods: the new semi-automated method using CiliarMove and the manual 
observation method. Each cluster of cells was selected based on a high number of cells present and on mucus-free regions, where cilia could beat without being trapped by mucus or debris. Ciliary motility analysis was performed by the same operator throughout the study.

The regions of interest (ROIs) were selected manually by the observer and were chosen based on the larger areas of mucus-free cilia tips with the largest visible movements (as in OLM et al. [27]). CBP was qualitatively assessed by a trained technician. Special attention was given to lack of coordination between cilia, cilia beat amplitude, presence of rotating cilia from top-view movies and stiffness of beating in proximal or distal regions of cilia.

\section{New software development for CBF analysis}

The software CiliarMove was developed in C\# programming language and compiled in Visual Studio Express (Microsoft, Redmond, WA, USA) freeware. The programme can automatically study the cilium movement within a sequence of images in greyscale. It is possible to process up to 1024 frames from a sequence of images. The CBF results obtained by the software were always compared with the results from the manual observation method. The software is compatible with the Microsoft Windows operating system. To run it the user needs to choose a sequence of images and then choose a personalised ROI, comprising a region where the beating cilia can be observed. It is possible to configure a desired conversion value for pixel size. To discover the frequency of the chosen ROI, the method calculates the fast Fourier transform (FFT) [28] for each pixel, which gives a set of frequencies with different amplitudes associated. After this, the software chooses the dominant frequency to represent each pixel. In addition, CiliarMove calculates an uncertainty associated to the frequency by using the fact that the result depends on the number of frames analysed, as indicated by the following equation.

$$
\text { Frequency resolution }(\mathrm{Hz})=\frac{\text { Recording speed (frames per second) }}{N \text { (number of frames) }}
$$

Each ROI frequency was compared with the manual observation method.

In the manual observation method, the sequence of images was played back at 30 or 60 frames per second or frame by frame; $\mathrm{CBF}$ was calculated by the observer timing 10 individual cilia beat cycles using the following equation.

$$
\text { Frequency }(\mathrm{Hz})=\frac{\text { Recording speed (frames per second) }}{\text { Number of frames elapsed during } 10 \text { beating cycles }} \times 10
$$

Additionally, in the CiliarMove software the user can choose to verify the FFT output as a histogram associated to each pixel (figure 1a). In summary, the software returns 1) a heat map of frequencies from the chosen ROI (figure 1a,c); 2) a histogram and a table with the distribution of frequencies (figure 1d); and 3) the respective percentage for all the frequencies found (figure 1e). It is possible to focus on an interval of frequencies defined by the user; the higher percentage of frequencies was the one we considered for each cell cluster. Each subject was then characterised by the average value from the 10 cell clusters. The software allows the creation of a table with the number of clusters analysed, the ROI number from the sample, the filename, the size of ROI and average, median, mode, standard deviation, minimum, maximum, range and frequency resolution $(\mathrm{Hz})$. The new software analyses .tiff files, although it supports other formats such as .png, .bmp or .jpg. The software can reliably analyse cilia movement from either top or side views of ciliated cells and provide an output in a few seconds per cluster, compared to the several minutes needed per cluster using the conventional manual method (figure 1f,g).

\section{Statistical analysis}

The results were tabulated and for each subject and each method, we registered the maximum and the minimum $\mathrm{CBF}$ value and calculated the mean \pm SD for 10 different ROIs corresponding to the 10 cell clusters. For comparisons between methods, the values used per subject were the means from the 10 cell clusters.

A linear regression model was performed to correlate the results obtained from the two different approaches used in this study. A Mann-Whitney-Wilcoxon test, t-test and a Bland-Altman analysis [29] were used to compare the groups.

\section{Results}

A total of 510 videos were analysed from healthy controls and patients (170 from healthy controls, 160 from the PCD-excluded group and 180 from the PCD group). Examples of movies from ciliated nasal 

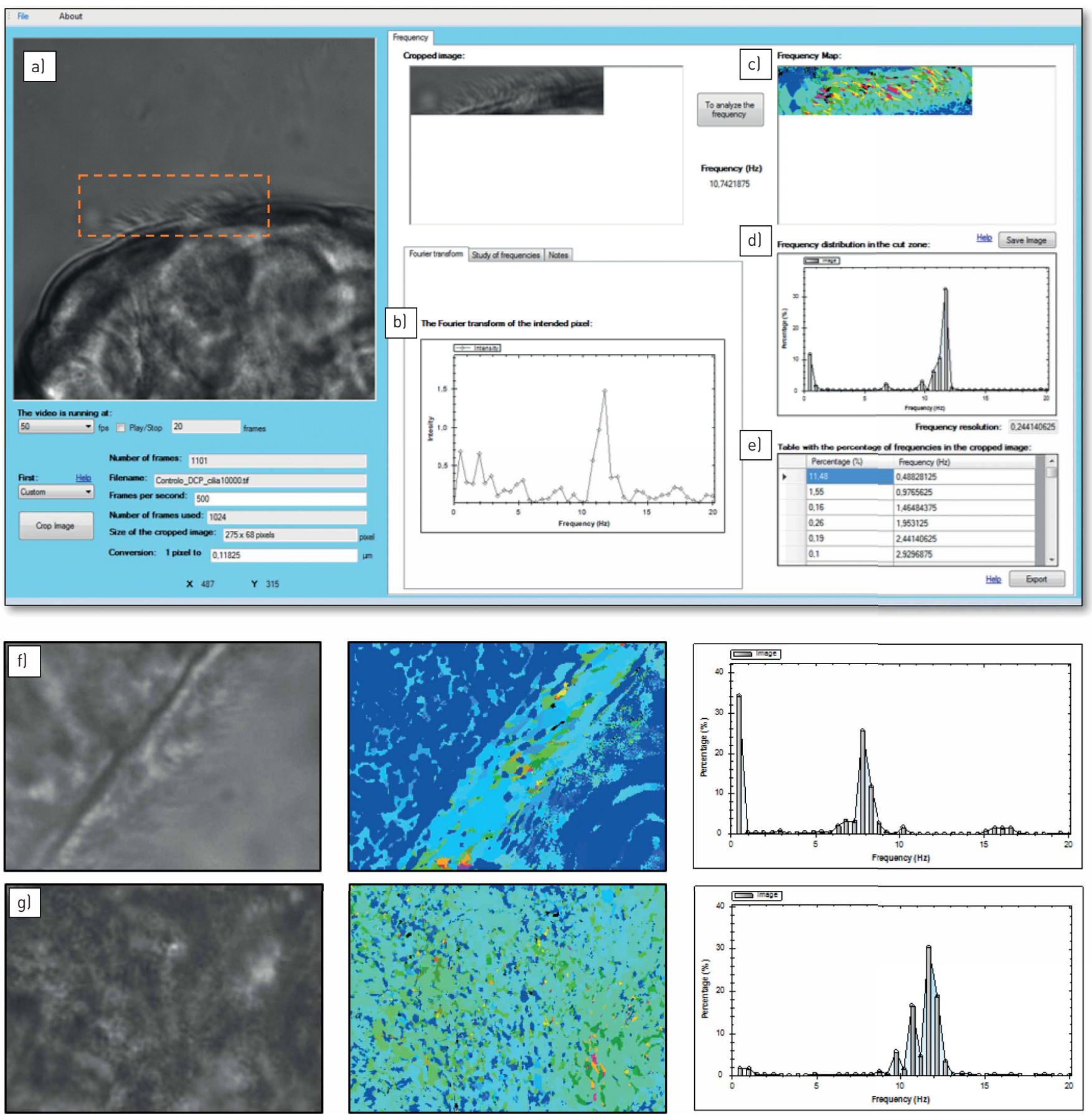

FIGURE 1 The CiliarMove software interface. The software was developed in C\# and works in the Microsoft Windows operating system. To run it the user needs to choose the sequence of images and then choose a personalised region of interest, as shown by the dotted red line in a). The different features of the frame sequence will be displayed below the movie: al video visualisation panel and "crop" feature; b) fast Fourier transform plot for a selected ("clicked") pixel; c) frequency heat map: the colour gradient used was dark blue for the lower frequencies, passing to green, yellow and finishing with red for higher frequencies; d) frequency histogram; e) table with values used in the histogram; f) example of a cropped side-view heat map and histogram; gl example of a cropped top-view heat map and histogram.

epithelial cells from the PCD group respective to different novel mutations can be found in the supplementary material (movies S1 for DNAH5, S2 for ZMYND10, S3 for CCDC40, S4 for DNAH11 and S5 for CCDC151).

All healthy control subjects had normal CBP based on the lack of stiff, rotational or uncoordinated cilia (see examples from a lateral view and top view in movies S6 and S7, respectively). According to 17 healthy control subjects, the CBF interval calculated using the conventional manual method (table 1 and figure 2) 


\section{TABLE 1 Detailed ciliary beat frequencies from the healthy control group}

\begin{tabular}{|c|c|c|c|c|c|}
\hline \multirow[t]{2}{*}{ Subject } & \multirow[t]{2}{*}{ Age years } & \multicolumn{4}{|c|}{ Ciliary beat frequency $\mathrm{Hz}$} \\
\hline & & Mean & Standard deviation & Minimum & Maximum \\
\hline 1 & 28 & $7.79 / 7.72$ & $2.1 / 2.23$ & $5.23 / 4.88$ & $11.52 / 11.72$ \\
\hline 2 & 23 & $6.99 / 6.84$ & $1.94 / 2.06$ & $5.42 / 4.88$ & $12.20 / 12.21$ \\
\hline 3 & 28 & $7.21 / 7.18$ & $1.29 / 1.38$ & $5.76 / 5.86$ & $10.04 / 10.25$ \\
\hline 4 & 35 & $6.97 / 6.88$ & $0.77 / 0.81$ & $5.52 / 5.37$ & $7.80 / 7.81$ \\
\hline 5 & 24 & $7.57 / 7.47$ & $1.24 / 1.30$ & $5.77 / 5.86$ & $9.65 / 9.77$ \\
\hline 6 & 27 & $6.99 / 7.03$ & $1.17 / 1.32$ & $5.37 / 4.88$ & $9.22 / 9.28$ \\
\hline 7 & 28 & $8.47 / 8.55$ & $1.05 / 1.11$ & $7.01 / 6.84$ & $10.44 / 10.74$ \\
\hline 8 & 30 & $7.99 / 7.91$ & $1.17 / 1.30$ & $6.27 / 5.86$ & $9.38 / 9.77$ \\
\hline 9 & 26 & $7.46 / 7.42$ & $0.84 / 0.80$ & $5.81 / 5.86$ & $8.80 / 8.79$ \\
\hline 10 & 25 & $7.62 / 7.59$ & $1.32 / 1.31$ & $5.63 / 5.37$ & $10.00 / 9.77$ \\
\hline 11 & 24 & $9.17 / 9.13$ & $1.87 / 2.03$ & $6.83 / 6.35$ & $13.48 / 13.67$ \\
\hline 12 & 28 & 7.33/7.32 & $0.99 / 1.10$ & $6.20 / 5.86$ & $9.19 / 9.28$ \\
\hline 13 & 28 & $7.97 / 7.96$ & $1.18 / 1.32$ & $5.87 / 5.86$ & $10.31 / 10.74$ \\
\hline 14 & 27 & $7.45 / 7.57$ & $1.48 / 1.58$ & $5.83 / 5.86$ & $10.53 / 10.74$ \\
\hline 15 & 23 & $8.52 / 8.55$ & $1.10 / 1.22$ & $6.75 / 6.35$ & $10.27 / 10.25$ \\
\hline 16 & 42 & $6.18 / 6.10$ & $0.73 / 0.77$ & $4.92 / 4.88$ & $7.24 / 7.32$ \\
\hline 17 & 43 & $7.00 / 6.94$ & $1.00 / 1.05$ & $5.85 / 5.86$ & $8.45 / 8.30$ \\
\hline
\end{tabular}

Data are presented as conventional/CiliarMove methods, unless otherwise stated.

varied from a minimum of $6.18 \mathrm{~Hz}$ to a maximum of $9.17 \mathrm{~Hz}$ (average of $7.57 \mathrm{~Hz}$ ) and the CiliarMove method ranged from 6.10 to $9.13 \mathrm{~Hz}$ (average of $7.54 \mathrm{~Hz}$ ). For the PCD-excluded group the values from the conventional method varied between 6.84 and $10.93 \mathrm{~Hz}$ (average of $8.85 \mathrm{~Hz}$ ), whereas for the CiliarMove method values ranged from 6.98 to $11.04 \mathrm{~Hz}$ (average of $8.81 \mathrm{~Hz}$ ) (table 2), presenting significantly higher CBFs by both methods than the healthy control group ( $\mathrm{t}$-test $\mathrm{p}<0.05$ ). The PCD group presented values between 0 and $14.30 \mathrm{~Hz}$ by the conventional method (average of $5.29 \mathrm{~Hz}$ ), while CiliarMove software detected values from 0 to $14.34 \mathrm{~Hz}$ (average of $5.21 \mathrm{~Hz}$ ) (table 3).

From subjects who had PCD genetic testing (table 4) a comparison between the results by HVMA, TEM and genetics was performed. Specifically, the subjects who presented mutations causing ODA defects (DNAH5) showed dyskinetic and uncoordinated ciliary movement with CBFs lower than those presented

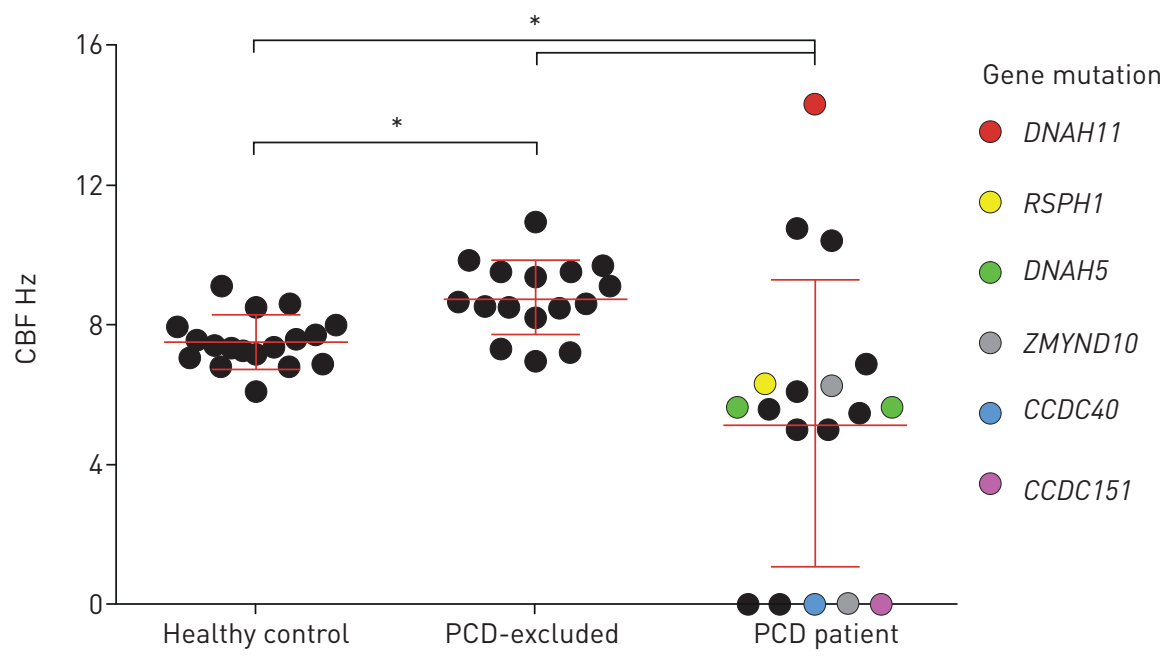

FIGURE 2 Ciliary beat frequency (CBF) measurements using the conventional manual method for the three different groups. Healthy control group $(n=17)$, primary ciliary dyskinesia (PCD)-excluded group $(n=16)$ and PCD group ( $n=18$ ). Each point indicates the mean CBF calculated for each subject. Coloured points represent the genetic result for the PCD mutation found in the subjects. Bars represent mean \pm SD. *: $p<0.05$ (t-test). 
TABLE 2 Detailed ciliary beat frequencies from the primary ciliary dyskinesia-excluded group

\begin{tabular}{|c|c|c|c|c|c|}
\hline \multirow[t]{2}{*}{ Subject } & \multirow[t]{2}{*}{ Age years } & \multicolumn{4}{|c|}{ Ciliary beat frequency $\mathrm{Hz}$} \\
\hline & & Mean & Standard deviation & Minimum & Maximum \\
\hline 1 & 10 & $10.93 / 11.04$ & $1.66 / 1.58$ & $8.55 / 8.3$ & $13.77 / 13.67$ \\
\hline 2 & 9 & $8.74 / 8.59$ & $1.91 / 1.77$ & $6.49 / 6.84$ & $12.25 / 13.67$ \\
\hline 3 & 36 & $9.44 / 9.42$ & $2.85 / 2.66$ & $6.27 / 6.34$ & $15.2 / 14.65$ \\
\hline 4 & 10 & $6.84 / 6.98$ & $0.77 / 0.80$ & $5.77 / 5.86$ & $8.25 / 8.30$ \\
\hline 5 & 16 & $8.22 / 8.25$ & $1.15 / 1.23$ & $6.35 / 6.35$ & $9.62 / 9.77$ \\
\hline 6 & 21 & $9.50 / 9.57$ & $0.90 / 0.93$ & $8.42 / 8.30$ & $11.06 / 11.23$ \\
\hline 7 & 4 & $8.56 / 8.64$ & $1.21 / 1.22$ & $6.97 / 6.83$ & $10.20 / 10.25$ \\
\hline 8 & 3 & $9.60 / 9.52$ & $1.64 / 1.22$ & $7.41 / 7.32$ & $12.2 / 11.72$ \\
\hline 9 & 4 & $7.40 / 7.37$ & $0.89 / 0.81$ & $5.96 / 6.34$ & $8.77 / 8.78$ \\
\hline 10 & 9 & $10.00 / 9.86$ & $0.97 / 0.85$ & $8.49 / 8.79$ & $11.66 / 11.23$ \\
\hline 11 & 15 & $8.83 / 8.55$ & $2.48 / 2.66$ & $5.99 / 5.86$ & $13.16 / 13.18$ \\
\hline 12 & 17 & $9.44 / 9.18$ & $2.22 / 2.23$ & $6.89 / 6.84$ & $14.49 / 14.65$ \\
\hline 13 & 14 & $9.58 / 9.67$ & $1.98 / 1.85$ & $6.54 / 6.84$ & $13.48 / 12.21$ \\
\hline 14 & 15 & $7.27 / 7.23$ & $1.14 / 1.19$ & $5.04 / 4.88$ & $8.47 / 8.30$ \\
\hline 15 & 8 & $8.67 / 8.60$ & $1.49 / 1.48$ & $5.93 / 5.86$ & $11.34 / 11.23$ \\
\hline 16 & 3 & $8.57 / 8.53$ & $1.89 / 1.80$ & $5.68 / 6.30$ & $12.14 / 12.20$ \\
\hline
\end{tabular}

by the healthy control and the PCD-excluded group (subject 2189935 mean $5.74 \mathrm{~Hz}$; subject 2255918 mean $5.83 \mathrm{~Hz}$ ), or presented complete absence of ciliary movement (CCDC151).

ZMYND10 is known to be involved in the pre-assembly of dynein arms [19], and mutations in ZMYND10 either led to total absence of ciliary movement (subject 1880724) or uncoordinated and reduced beating amplitude (subject 2014246), showing CBFs within our healthy control group range. The mutation in subject 2014246, although previously reported, had no description of CBF or CBP [30]. Interestingly, while TEM on samples from subject 1880724 showed absence of both IDA and ODA with no CBF detected, results from subject 2014246 had normal TEM and CBF within normal ranges (minimum 5.02/4.39 Hz and maximum 8.90/9.04 Hz, according to conventional and CiliarMove methods, respectively) and agreeing with that reported by ZARIWALA et al. [30].

As reported previously [31], the mutation we found in DNAH11 led to high CBF values using both methods (average 14.30/14.34 Hz according to conventional and CiliarMove methods, respectively; table 3), which is above the CBF average for both control groups: the healthy control group and PCD-excluded group. Additionally, we detected a reduction in basal ciliary amplitude and hyperkinetic edges (supplementary movie S4).

In addition, the novel allele for CCDC40 described for subject 2009075 led to a severe impairment of motility with presence of static cilia or residual motion (supplementary movie S4), despite the fact that the sample was not conclusive for TEM. In contrast, defects on the central pair apparatus caused by a

TABLE 3 Detailed ciliary beat frequencies from the primary ciliary dyskinesia group

\begin{tabular}{|c|c|c|c|c|c|}
\hline \multirow[t]{2}{*}{ Subject } & \multirow[t]{2}{*}{ Age years } & \multicolumn{4}{|c|}{ Ciliary beat frequency $\mathrm{Hz}$} \\
\hline & & Mean & Standard deviation & Minimum & Maximum \\
\hline 2189935 & 10 & $5.74 / 5.70$ & $1.12 / 1.07$ & $3.33 / 3.42$ & $6.94 / 7.32$ \\
\hline 1880724 & 4 & 0 & 0 & 0 & 0 \\
\hline 2255918 & 16 & $5.83 / 5.71$ & $1.70 / 1.78$ & $4.17 / 3.9$ & $9.06 / 9.28$ \\
\hline 2009075 & 9 & 0 & 0 & 0 & 0 \\
\hline 2310483 & 15 & $6.42 / 6.35$ & $0.79 / 0.86$ & $5.56 / 5.37$ & $7.86 / 7.81$ \\
\hline 2438548 & 10 & $14.30 / 14.34$ & $2.26 / 2.33$ & $9.04 / 8.90$ & $17.18 / 17.09$ \\
\hline 2014246 & 5 & $6.29 / 6.27$ & $1.36 / 1.25$ & $5.02 / 4.39$ & $8.90 / 9.04$ \\
\hline 2137214 & 12 & 0 & 0 & 0 & 0 \\
\hline
\end{tabular}




\section{TABLE 4 Genetic and transmission electron microscopy (TEM) findings from eight subjects with primary ciliary dyskinesia}

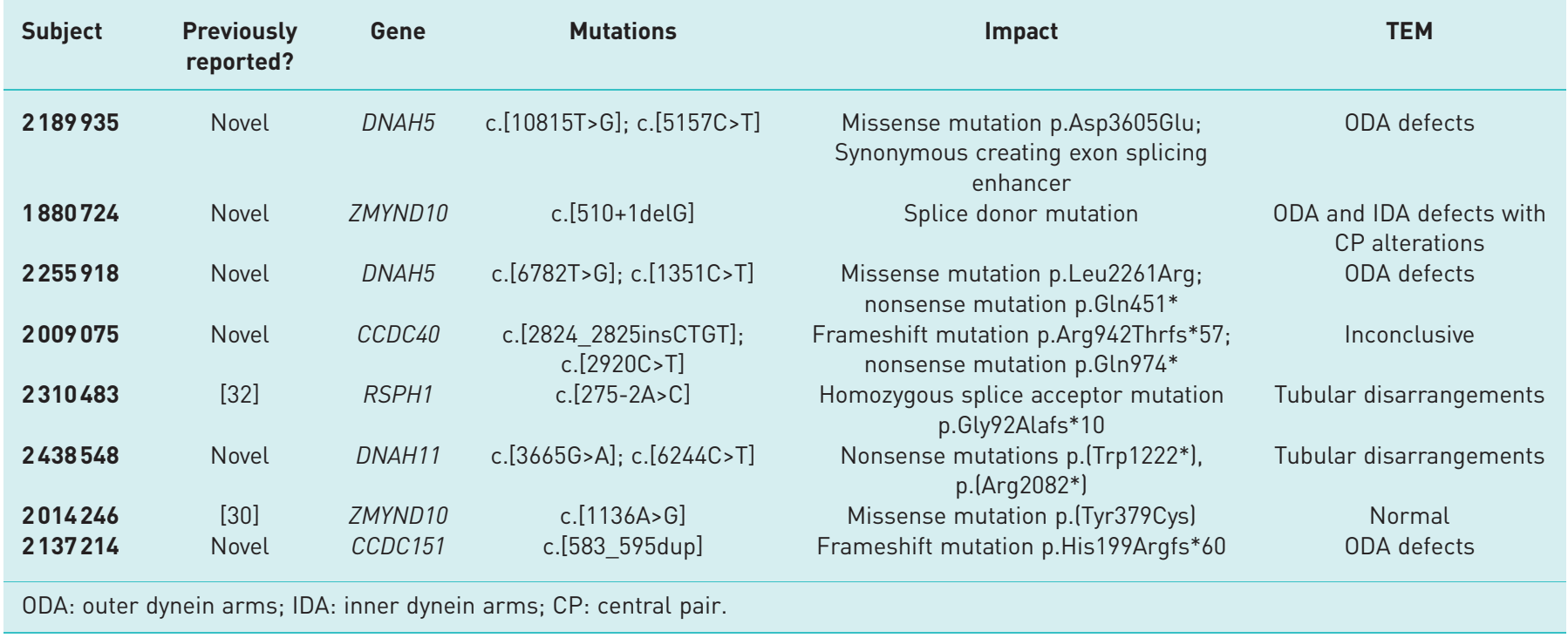

previously described genetic variant $R S P H 1$ (table 4) led to a normal range of CBF compared with our control groups, but cilia showed reduced beating amplitude and stiffer movement, as described previously [32], highlighting the importance of CBP evaluation.

In summary, when comparing the $\mathrm{CBF}$ interval values from the healthy control group with those from the PCD group, we identified $78 \%$ (14 out of 18 subjects) of the PCD-affected subjects, while the comparison of the PCD-excluded group range of values with those from the PCD group identified $89 \%$ of the PCD-affected subjects (16 out of 18 subjects).

Next, for better validating the new software on measuring frequencies of moving cilia we excluded the PCD subjects with immotile cilia from the comparison. Therefore, 460 movies were re-analysed and provided CBF values between 5.16 and $14.3 \mathrm{~Hz}$. We then compared these values to the ones obtained with the conventional method (table 3 and figure 2). For better characterisation we calculated the linear correlation as $\mathrm{R}^{2}=0.9895$ (figure 3a), indicating a high degree of association between the two methods. Additionally, we performed a Bland-Altman analysis for assessing the agreement between the two
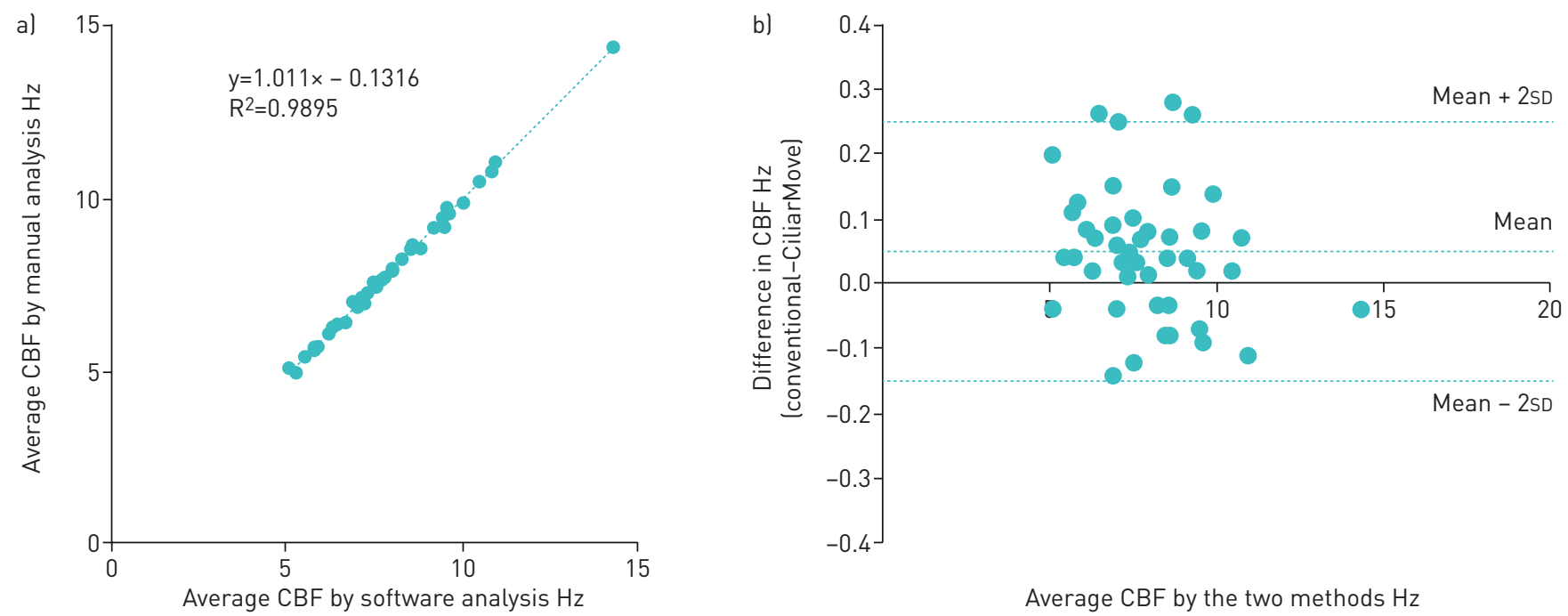

FIGURE 3 Comparison of ciliary beat frequency (CBF) calculation methods: visual manual analysis and CiliarMove software. a) Linear regression of the mean CBF measurements comparing the two methods; b) Bland-Altman plot of the mean CBF measurements comparing the two methods. The results are in the range of the average difference of $\pm 2 \mathrm{SD}$. 
methods (figure 3b) [33]. For 95\% limits of agreement, we obtained a mean \pm SD difference of $0.05 \pm .01 \mathrm{~Hz}$. These results further show that the overall averages of both sets of measurements are nearly identical.

Furthermore, as the software relies on FFT to perform CBF calculation on broad ciliated regions, it eliminates systematic and random errors that the conventional manual method may not account for, as it depends on the analyser. Thus, we consider the new software CiliarMove to be validated and suitable for $\mathrm{CBF}$ measurement for improved, faster and unbiased PCD diagnostic procedure.

\section{Discussion}

To the best of our knowledge, this is the first study to date comparing values of ciliary function of nasal epithelial cells between a Portuguese healthy group and a PCD patient group. We determined that the $\mathrm{CBF}$ range at $25^{\circ} \mathrm{C}$ (for a control group of 17 adult healthy volunteers) was $6.18-9.17 \mathrm{~Hz}$ (table 1).

When we compared our results to the study done by RAIDT et al. [19], which was performed at the same temperature, for a German group of 10 adult healthy controls, we can conclude they identified a wider range of CBF, from $4.25 \mathrm{~Hz}$ to $11.63 \mathrm{~Hz}$. We hypothesise that a reason for this difference between studies might be population genetic variability. This supports the need for each diagnostic centre to assess its own range of $\mathrm{CBF}$ in groups of healthy volunteers. Alternatively, our findings may reflect sample size discrepancies, slight alterations in lab methodology or age of participants. The association between age and $\mathrm{CBF}$ in humans has been investigated in insufficient studies to confirm a correlation between the two variables [34-37]. Even so, some evidence supports that individuals aged $>40$ years present slower CBF and decreased mucociliary clearance $[35,36]$. Taking this into consideration, it is important to stress that our healthy control group subjects were mostly aged $<40$ years.

Regarding the power of $\mathrm{CBF}$ alone as a diagnostic measure for PCD our data showed that for higher frequencies, i.e. caused by variants in DNAH11, or for very low frequencies, caused by variants in ZMYND10, CCDC40 or CCDC151, CBF was sufficient to detect a PCD subject. However, for intermediate-range frequencies, the statistical difference between the healthy control group range and the range from confirmed PCD subjects proved unreliable for correctly diagnosing the disease, detecting only $64 \%$ of the cases. Hence the importance of the CBP characterisation in the combined assessment of the ciliary function, as previously discussed and defined in several guidelines [13, 38].

The frequency range obtained for the PCD-excluded group presented significantly higher CBF values when compared to the healthy control group (figure 2). This observation goes against the trend reported by RAIDT et al. [19], which showed that the non-PCD group had lower CBF compared to healthy controls. Again, several factors may be at the origin of this divergence. One of the reasons may be due to different eligibility criteria for the PCD-excluded group. While we included all PCD-negative patients who had both upper and lower respiratory symptoms (e.g. bronchiectasis and chronic sinusitis), the German group did not. This variability can be summarised by different respiratory pathogens in place, different inflammatory responses and different therapeutic approaches in action for each group.

Numerous studies have documented the ability of bacterial toxins, as well as inflammatory mediators and pharmacological agents in modelling of CBF [39-43]. Without a deep understanding of the specific conditions of these patients and how the management of the disease is being carried out in each clinical centre, it is difficult to ascertain the real reasons for such differences. Therefore, it is imperative that diagnostic centres converge on a consensual system for standardising the selection of the appropriate control CBF groups.

Regarding the new software here successfully validated, there are two previously developed computer programmes similar to CiliarMove. The most recent one is named ciliaFA [44] and is also freely available. ciliaFA is as an open-source ImageJ plugin. It determines the average pixel intensity of up to $40 \times 40$ ROI per frame. The ciliaFA plugin exports a dataset of up to 1600 ROI to Excel 2007 (Microsoft) where a basic macro is initiated to perform the FFT analysis. The complex number is translated using an Excel function and the dominant frequency within the range is then established. Thus, ciliaFA requires two other software programmes to retrieve the final result, proving to be less practical than CiliarMove, which allows all the results to be achieved with the same software. However, ciliaFA performs noise reduction, which we did not incorporate into CiliarMove. Additionally, Sisson-Ammons Video Analysis (SAVA), built in 2003, is still much in use [45]. SAVA is commercial software and a digital system which samples relatively small pixel areas at 85-100 fps, allowing measurement of CBF up to a maximum frequency of $42.5 \mathrm{~Hz}$, which means that SAVA is tailored to analysis of nasal cilia frequency. For a research laboratory that investigates higher cilia beat frequencies registered in some animal models, SAVA may be limited. In contrast, CiliarMove is open-source and processes all the pixels selected in $\geqslant 1024$ frames, enabling CBF measurements at $500 \mathrm{fps}$, as suggested by the PCD guidelines [13]. 
In addition, the user-friendly interface in CiliarMove allows poorly trained users to perform the evaluation of the CBF easily, as witnessed in our lab when training students during small rotational studies.

Moreover, our software offers a potential cost benefit when compared to the widely used SAVA system. The fact that CiliarMove can be easily adapted to any high-speed image equipment allows the construction of low-budget HVMA setups. As shown by CHEN et al. [46], the HVMA cost for CBF measurement can be reduced to the price of a consumer-grade cellphone camera, which is far below the SAVA system market value (EUR 3000-7000 [47]). Therefore, our software can provide a valid solution to centres in developing countries that could not otherwise afford to implement this diagnostic test. One potential disadvantage of the CiliarMove software is that it only allows $1.5 \mathrm{~GB}$ of computer's random access memory to be used.

More recently, another software programme based on using algorithms from computer vision and machine learning was developed by QuinN et al. [48] to measure cilia motion. This software represents a step forward in the quantification of $\mathrm{CBP}$, which is one of the most discriminatory parameters for the PCD diagnostics. QuINN et al.'s [48] recent software also includes CBF evaluation, and could thus provide an all-in-one pipeline. Unfortunately, although initially conceived to be provided as a web service, this software is still not freely available to the research community.

The limitations of our study are the small sample size, meaning it is probably not entirely representative of the Portuguese population, and the fact that we could only analyse adult healthy controls despite diagnosis being mostly (and preferably) done in children. The implementation of the diagnosis of PCD in Portugal according to the European guidelines, took place 7 years ago and has since grown significantly [49], leading us to predict that further studies will be undertaken in the coming years.

We conclude that computerised $\mathrm{CBF}$ analysis using CiliarMove represents an advantage because it correlates highly with standard visual manual methods, with the advantage that it remarkably speeds up data analysis, which is extremely useful in a diagnostic scenario. Most importantly, we have shown here that CiliarMove can help to better characterise previously reported or novel PCD genetic variants, by providing a range of CBF values per subject that is highly unbiased.

Acknowledgements: S.S. Lopes, C. Constant, L. Pereira, A. Pinto and P. Sampaio were members of COST Action BEAT-PCD (BM1407). The authors want to thank all the volunteers who allowed this study to be performed and the PIATI programme organised by the NOVA Medical School students' union and to the project LysoCil funded by the European Union Horizon 2020 research and innovation under grant agreement No 811087.

Ethics statement: This study was approved by the ethics committee of the NOVA Medical School. Informed consent was obtained from all healthy volunteers and patients.

Availability of data and material: All data generated or analysed during this study are included in this published article. The software CiliarMove is deposited in the Zenodo repository as: Mónica Ferro da Silva, Pedro Sampaio, Inês Vale, Mónica Roxo-Rosa, Carla Quintão, Carolina Constant and Susana Santos Lopes. (2017, August 29). CiliarMove software. Zenodo. http://doi.org/10.5281/zenodo.853251

Author contributions: P. Sampaio performed all the experiments and data analysis, and wrote the manuscript; M.F. da Silva performed and analysed experiments with healthy controls; I. Vale and C.M. Quintão designed and produced the CiliarMove programme; M. Roxo-Rosa revised the data analysis and the manuscript; C. Constant and L. Pereira analysed patient clinical history, coordinated nasal nitric oxide analyses from the subjects and provided the nasal brushings. A. Pinto performed transmission electron microscopy from the nasal samples taken from patients. S.S. Lopes designed and supervised the study and wrote the manuscript.

Support statement: This study was supported by the Fundação para a Ciência e a Tecnologia (PTDC/BEXBID/1411/ 2014 research grant). S.S. Lopes was funded by FCT Investigator IF/00951/2012, by NOVA Medical School and by FCT CEEC-IND 2018. P. Sampaio was funded by the PhD fellowship FCT: SFRH/BD/111611/2015. M. Roxo-Rosa was funded by the UID/Multi/04462/2013-LISBOA-01-0145-FEDER-007344 grant (iNOVA4Health). C.M. Quintão was funded by Fundação para a Ciência e Tecnologia (UID/FIS/04559/2013). S.S. Lopes participates in and acknowledge financial support from the COST Action BEAT-PCD (BM1407). S.S. Lopes received funding from project LysoCil funded by the European Union Horizon 2020 research and innovation under grant agreement No 811087. Funding information for this article has been deposited with the Crossref Funder Registry.

Conflict of interest: None declared.

\section{References}

1 Rubbo B, Lucas JS. Clinical care for primary ciliary dyskinesia: current challenges and future directions. Eur Respir Rev 2017; 26: 170023.

2 O'Callaghan C, Chetcuti P, Moya E. High prevalence of primary ciliary dyskinesia in a British Asian population. Arch Dis Child 2010; 95: 51-52.

3 Ellerman A, Bisgaard H. Longitudinal study of lung function in a cohort of primary ciliary dyskinesia. Eur Respir $J$ 1997; 10: 2376-2379.

4 Lucas JS, Burgess A, Mitchison HM, et al. Diagnosis and management of primary ciliary dyskinesia. Arch Dis Child 2014; 99: 850-856. 
5 Shapiro AJ, Zariwala MA, Ferkol T, et al. Diagnosis, monitoring, and treatment of primary ciliary dyskinesia: PCD foundation consensus recommendations based on state of the art review. Pediatr Pulmonol 2016; 51: 115-132.

6 Sironen A, Shoemark A, Patel M, et al. Sperm defects in primary ciliary dyskinesia and related causes of male infertility. Cell Mol Life Sci 2020; 77: 2029-2048.

7 Horani A, Ferkol TW, Dutcher SK, et al. Genetics and biology of primary ciliary dyskinesia. Paediatr Respir Rev 2016; 18: 18-24.

8 Badano JL, Mitsuma N, Beales PL, et al. The ciliopathies: an emerging class of human genetic disorders. Annu Rev Genomics Hum Genet 2006; 7: 125-148.

9 Papon JF, Coste A, Roudot-Thoraval F, et al. A 20-year experience of electron microscopy in the diagnosis of primary ciliary dyskinesia. Eur Respir J 2010; 35: 1057-1063.

10 Lin J, Tritschler D, Song K, et al. Building blocks of the nexin-dynein regulatory complex in Chlamydomonas flagella. J Biol Chem 2011; 286: 29175-29191.

11 Porter ME. Flagellar motility and the dynein regulatory complex. In: King, SM, ed. Dyneins. London, Academic Press, 2012; pp. 336-365.

12 Behan L, Dimitrov BD, Kuehni CE, et al. PICADAR: a diagnostic predictive tool for primary ciliary dyskinesia. Eur Respir J 2016; 47: 1103-1112.

13 Lucas JS, Barbato A, Collins SA, et al. European Respiratory Society guidelines for the diagnosis of primary ciliary dyskinesia. Eur Respir J 2017; 49: 1601090.

14 Sousa R, Constant C, Bandeira T, et al. Primary ciliary dyskinesia: updates on diagnosis, follow-up and treatment. Port J Pediatr 2018; 49: 342-349.

15 Damseh N, Quercia N, Rumman N, et al. Primary ciliary dyskinesia: mechanisms and management. Appl Clin Genet 2017; 10: 67-74.

16 Boon M, Smits A, Cuppens H, et al. Primary ciliary dyskinesia: critical evaluation of clinical symptoms and diagnosis in patients with normal and abnormal ultrastructure. Orphanet J Rare Dis 2014; 9: 11.

17 Bush A, Cole P, Hariri M, et al. Primary ciliary dyskinesia: diagnosis and standards of care. Eur Respir J 1998; 12 982-988.

18 Blanchon S, Legendre M, Bottier M, et al. Deep phenotyping, including quantitative ciliary beating parameters, and extensive genotyping in primary ciliary dyskinesia. J Med Genet 2020; 57: 237-244.

19 Raidt J, Wallmeier J, Hjeij R, et al. Ciliary beat pattern and frequency in genetic variants of primary ciliary dyskinesia. Eur Respir J 2014; 44: 1579-1588.

20 Papon J-F, Bassinet L, Cariou-Patron G, et al. Quantitative analysis of ciliary beating in primary ciliary dyskinesia: a pilot study. Orphanet J Rare Dis 2012; 7: 78.

21 Stannard WA, Chilvers MA, Rutman AR, et al. Diagnostic testing of patients suspected of primary ciliary dyskinesia. Am J Respir Crit Care Med 2010; 181: 307-314.

22 Rubbo B, Shoemark A, Jackson CL, et al. Accuracy of high-speed video analysis to diagnose primary ciliary dyskinesia. Chest 2019; 155: 1008-1017.

23 Coraux C, Hajj R, Lesimple $\mathrm{P}$, et al. In vivo models of human airway epithelium repair and regeneration. Eur Respir Rev 2005; 14: 131-136.

24 Dupuit F, Gaillard D, Hinnrasky J, et al. Differentiated and functional human airway epithelium regeneration in tracheal xenografts. Am J Physiol Cell Mol Physiol 2000; 278: L165-L176.

25 Shoemark A, Boon M, Brochhausen C, et al. International consensus guideline for reporting transmission electron microscopy results in the diagnosis of primary ciliary dyskinesia (BEAT PCD TEM Criteria). Eur Respir J 2020; 55: 1900725

26 Fassad MR, Patel MP, Shoemark A, et al. Clinical utility of NGS diagnosis and disease stratification in a multiethnic primary ciliary dyskinesia cohort. J Med Genet 2020; 57: 322-330.

27 Olm MAK, Kögler JE, Macchione M, et al. Primary ciliary dyskinesia: evaluation using cilia beat frequency assessment via spectral analysis of digital microscopy images. J Appl Physiol 2011; 111: 295-302.

28 Kim W, Han TH, Kim HJ, et al. An automated measurement of ciliary beating frequency using a combined optical flow and peak detection. Healthc Inform Res 2011; 17: 111-119.

29 Bland JM, Altman DG. Statistical methods for assessing agreement between two methods of clinical measurement Int J Nurs Stud 2010; 47: 931-936.

30 Zariwala MA, Gee HY, Kurkowiak M, et al. ZMYND10 is mutated in primary ciliary dyskinesia and interacts with LRRC6. Am J Hum Genet 2013; 93: 336-345.

31 Schwabe GC, Hoffmann K, Loges NT, et al. Primary ciliary dyskinesia associated with normal axoneme ultrastructure is caused by DNAH11 mutations. Hum Mutat 2008; 29: 289-298.

32 Kott E, Legendre M, Copin B, et al. Loss-of-function mutations in RSPH1 cause primary ciliary dyskinesia with central-complex and radial-spoke defects. Am J Hum Genet 2013; 93: 561-570.

33 Giavarina D. Understanding Bland Altman analysis. Biochem Med 2015; 25: 141-151.

34 Jorissen M, Willems T, Van der Schueren B. Nasal ciliary beat frequency is age independent. Laryngoscope 1998 108: 1042-1047.

35 Ho JC, Chan KN, Hu WH, et al. The effect of aging on nasal mucociliary clearance, beat frequency, and ultrastructure of respiratory cilia. Am J Respir Crit Care Med 2001; 163: 983-988.

36 Svartengren M, Falk R, Philipson K. Long-term clearance from small airways decreases with age. Eur Respir J 2005; 26: 609-615.

37 Bailey KL, Bonasera SJ, Wilderdyke M, et al. Aging causes a slowing in ciliary beat frequency, mediated by PKC Am J Physiol Cell Mol Physiol 2014; 306: L584-L589.

38 Shapiro AJ, Davis SD, Polineni D, et al. Diagnosis of primary ciliary dyskinesia. An official American Thoracic Society clinical practice guideline. Am J Respir Crit Care Med 2018; 197: e24-e39.

39 Merkus P, Romeijn SG, Verhoef JC, et al. Classification of cilio-inhibiting effects of nasal drugs. Laryngoscope 2001; 111: 595-602.

40 Hofmann T, Gugatschga M, Koidl B, et al. Influence of preservatives and topical steroids on ciliary beat frequency in vitro. Arch Otolaryngol Neck Surg 2004; 130: 440-445.

41 Mallants R, Jorissen M, Augustijns P. Beneficial effect of antibiotics on ciliary beat frequency of human nasal epithelial cells exposed to bacterial toxins. J Pharm Pharmacol 2008; 60: 437-443. 
42 Gudis D, Zhao K-Q, Cohen NA. Acquired cilia dysfunction in chronic rhinosinusitis. Am J Rhinol Allergy 2012; 26: 1-6.

43 Yaghi A, Zaman A, Cox G, et al. Ciliary beating is depressed in nasal cilia from chronic obstructive pulmonary disease subjects. Respir Med 2012; 106: 1139-1147.

44 Smith CM, Djakow J, Free RC, et al. ciliaFA: a research tool for automated, high-throughput measurement of ciliary beat frequency using freely available software. Cilia 2012; 1: 14 .

45 Sisson JH, Stoner JA, Ammons BA, et al. All-digital image capture and whole-field analysis of ciliary beat frequency. J Microsc 2003; 211: 103-111.

46 Chen JJ, Lemieux BT, Wong BJF. A low-cost method of ciliary beat frequency measurement using iPhone and MATLAB: rabbit study. Otolaryngol Head Neck Surg 2016; 155: 252-256.

47 Kouis P, Papatheodorou SI, Middleton N, et al. Cost-effectiveness analysis of three algorithms for diagnosing primary ciliary dyskinesia: a simulation study. Orphanet J Rare Dis 2019; 14: 142.

48 Quinn SP, Zahid MJ, Durkin JR, et al. Automated identification of abnormal respiratory ciliary motion in nasal biopsies. Sci Transl Med 2015; 7: 299ra124.

49 Carolina C, Rodrigo S, Andreia P, et al. A new era in the diagnosis of primary ciliary dyskinesia. Port J Pediatr 2018; 49: 235-342. 